\title{
Isolated Facial Nerve Palsy in Childhood Acute Myeloid Leukemia
}

\author{
MG HAFIZ ${ }^{\mathrm{a}}$, A ISLAM ${ }^{\mathrm{b}}$, MA MANNAN ${ }^{\mathrm{c}}$, F RAHMAN $^{\mathrm{d}}$
}

\begin{abstract}
Summary:
Case report-1: Sabarna, a female baby of fifteen months old was admitted in Pediatric Hematology and Oncology, Bangabandhu Sheikh Mujib Medical University (BSMMU), Dhaka, Bangladesh with the complaints of deviation of the angle of mouth to right side and inability to close the left eye for five days. Systemic examination revealed an incision scar mark on the left mastoid region. Facial asymmetry as evidenced by smooth forehead, absence of nasolabial fold, drooping of the angle of mouth and nonclosure of eyelid on left side. She was mildy pale, mild hepatomegaly, no lymphadenopathy, bone pain or bleeding manifestation. Physical, hematological and histopathological investigations the features were suggestive of facial nerve palsy due to granulocytic sarcoma (GS) with acute myeloid leukemia (AML). Following second day of chemotherapy her vital signs suddenly deteriorated and succumbed probably due to the leukostasis or intracranial hemorrhage.
\end{abstract}

Introduction:

Granulocytic sarcoma (GS) is an extramedullary tumor of granulocytic lineage that has a long recognized association with acute myeloid leukemia (AML). ${ }^{1}$ It usually occurs in patients with AML, myeloproliferative disorders or myelodysplasia but can develop in patients with no known hematologic disorder. ${ }^{2,3} \mathrm{GS}$, describes extramedullary collections of leukemic cells. These collections can occur, alebeit

a. Dr. Md. Golam Hafiz, MD, Assistant Professor, Paediatric Haematology and Oncology, Dept. of Paediatrics, BSMMU, Dhaka.

b. Dr. Afiqul Islam, FCPS, MD, Professor of Paediatric Haematology and Oncology, Dept. of Paediatrics, BSMMU, Dhaka.

c. Dr. MA Mannan, M.Phil (Edin), FCPS (BD), FCPS (Pak), FRCP (Edin), Professor of Paediatric Haematology and Oncology, Chairman, Dept. of Paediatrics, BSMMU, Dhaka.

d. Dr. Fazlur Rahman, MCPS, D-Card, MD, Associate Professor, Dept of Cardiology, BSMMU, Dhaka.

Address of Correspondence: Dr. Md. Golam Hafiz, MD, Assistan Professor, Paediatric Haematology and Oncology, Dept. of Paediatrics, BSMMU, Dhaka.

Received: 18 December, 2007

Accepted: 12 February, 2008
Case report-2: Chayon, another nine month old female baby was admitted in the same unit with the history of fever, proptosis of both eye balls, deviation of the angle of mouth to the right side and unable to close the left eye for one month. Physical examination revealed facial asymmetry with the features of left sided facial nerve palsy along with proptosis of eyeballs. She was moderately pale, moderate hepatosplenomegaly, no bleeding manifestation or bone pain. Physical, hematological and cerebrospinal fluid (CSF) examination the features were suggestive of facial nerve palsy due to leukemic cell infiltration with AML. Following induction and consolidation the baby is on continuation phase with regular follow up for last 9 month having significant improvement of her facial nerve palsy along with hematological remission.

(J Bangladesh Coll Phys Surg 2010; 28: 53-58)

rarely, as the sole evidence of leukemia. In a review of 3 AML, studies conducted by the former Children Cancer Group, $<1 \%$ of patients had isolated GS and $11 \%$ had GS along with marrow disease at the time of diagnosis. $^{4}$

GS is a localized extramedullary tumor composed of immature cells of the granulocytic series. Most cases of GS occur associated with acute or chronic leukemia or myeloproliferative disorders. ${ }^{5}$ Rarely the tumor may be observed before the diagnosis of any hematological malignancy ${ }^{3,6}$ and most of them are harbingers of existing or impending AML. GS may occur in almost every part of the body ${ }^{5,7}$ but the spinal cord invasion and symptomatic facial nerve palsy are relatively uncommon. ${ }^{8,9,10}$ It may be due to leukemic cell infiltration at any site of facial nerve or occurrence of GS in the temporal bone or auditory canal and may be accompanied by hearing loss. ${ }^{9}$ Symptomatic facial nerve involvement in AML patients is rare. 9,10 
The clinical picture of central nervous system (CNS) leukemia is usually one of the meningeal irritations with headache, vomiting, photophobia and the signs simply of raised intracranial pressure. Occasionally, fits can be the presenting feature or cranial nerve involvement, particularly isolated VII nerve palsies. There is also well recognized syndrome of hyperphagia, pathological weight gain and somnolence thought to be due to involvement of the hypothalamus. ${ }^{11}$

To the best of our knowledge no account of facial nerve palsy due to GS or leukemic cell infiltration has earlier been reported in Bangladesh with childhood AML. To highlight this issue, here we are reporting two cases of childhood AML with isolated facial nerve palsy.

\section{Case Report-1:}

Sabarna, a fifteen month old female child weighing $10 \mathrm{~kg}$ hailing from Bagerhat, Bangladesh was admitted in Pediatric Neurology, BSMMU on $3^{\text {rd }}$ September, 2007 with the complaints of deviation of the angle of mouth to right side and inability to close the left eye for five days (Fig-I). Before admission, she was seen by an otorhinolaryngologist because of a small mass in

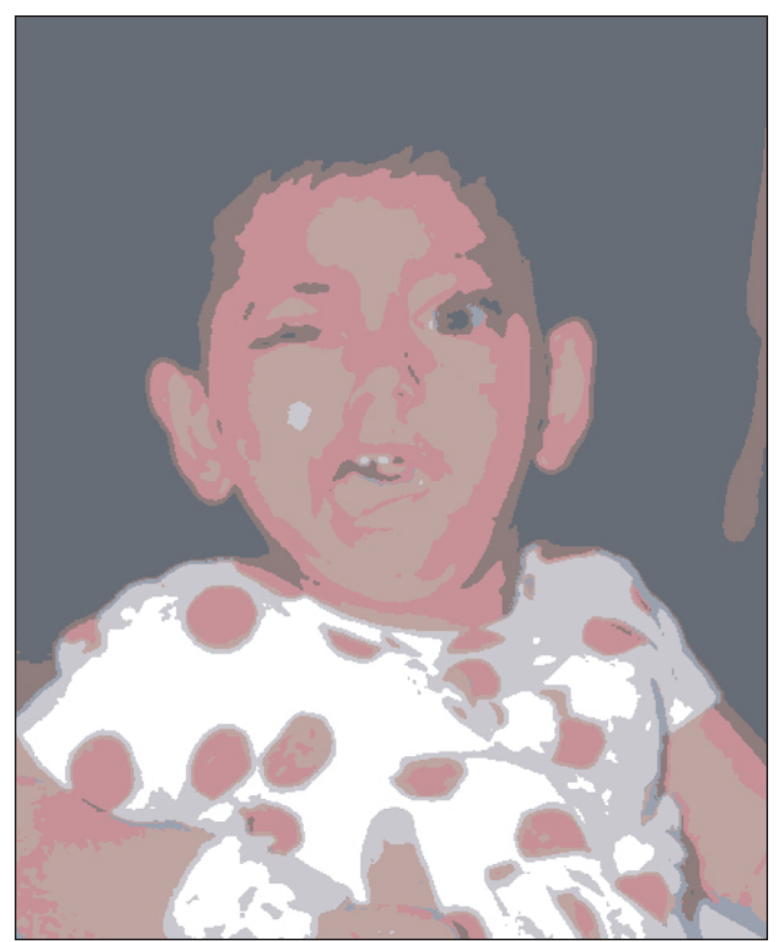

Fig.-1: Photograph (facial palsy) at presentation the auditory canal of the left ear. The mass lesion was excised totally to obtain decompression but there was no significant improvement of her complaints. The patient came of an average socioeconomic background, completely immunized and her general condition was good. There was no history of fever, vomiting, bone pain, cough, respiratory distress, bleeding manifestation, neck swelling, ear discharge, jaw pain, any trauma to head or blood transfusion. She was delivered at home at term without any complication. Milestone of development up to this time was appropriate for her age.

On systemic examination, there was an incision scar mark on the left mastoid region. The child was conscious, afebrile, restless, facial asymmetry as evidenced by smooth forehead, absence of nasolabial fold, drooping of the angle of mouth and non-closure of eyelid on left side. She was a mildly pale, skin survey revealed normal, no lymphadenopathy but mild hepatomegaly was present. Pupillary examination findings were normal. She was then treated with dexamethasone and facial massage having no significant improvement. Histopathological examination of the tumor mass showed various mature and immature granular cells with large mononuclear cells which had lobulated nucleus and immature loose structure of chromatin which was consistent with GS (Fig-2). Then, she was transferred to Pediatric Hematology and Oncology, BSMMU for further evaluation.

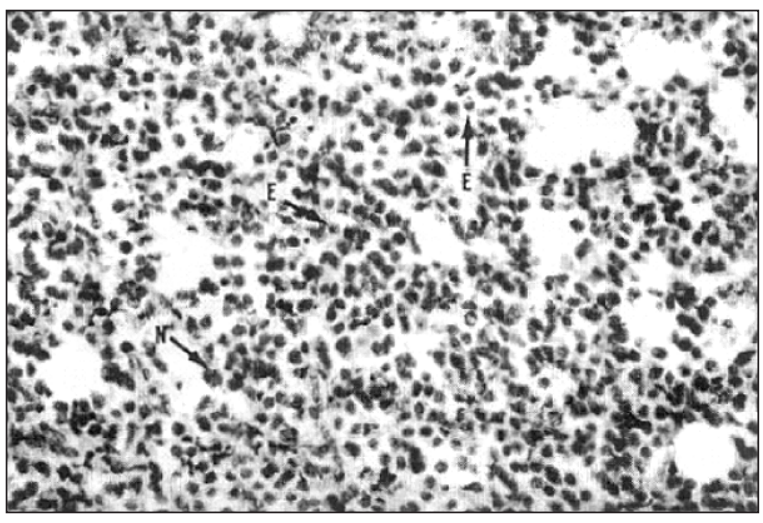

Fig.-2: Histopathologically the tumor is composed of a relatively uniform population of immature cells. There are some immature eosinophils (E) and neutrophils $(N)$

Hematological investigations showed the followings: hemoglobin level $8.2 \mathrm{gm} / \mathrm{dl}$, ESR $35 \mathrm{~mm}$ after first hour (westergren method), total leukocyte count 300 x $10^{9} / \mathrm{L}$, platelet count $40 \times 10^{9} / \mathrm{L}$, differential count: 
neutrophil $10 \%$, lymphocytes $30 \%$ and myeloblast $60 \%$. Peripheral blood film analysis showed the following: red blood cell showed anisochromia with anisocytosis, white blood cell showed shift to the left with the prominence of myeloblast and platelet were reduced. CSF study was absence of leukemic cell. Bone marrow morphology and cytochemistry were as follows: hypercellular marrow with increased myeloid erythroid ratio. Erythopoesis was depressed and granulopoesis was hyperplastic and showed more than $30 \%$ blasts having large nucleus with abundant cytoplasm containing azurophilic granules. Nucleus contains multiple prominent nucleoli with loose chromatin material. Megakaryocytes were reduced. Cytochemistry of the aspirated bone marrow showed periodic acid-Schiff (PAS) stain and myeloperoxidase (MPO) stain were positive and diagnosed as AML$\mathrm{M}_{4}$ according to French-American-British (FAB) classification (Fig-3 \& 4).

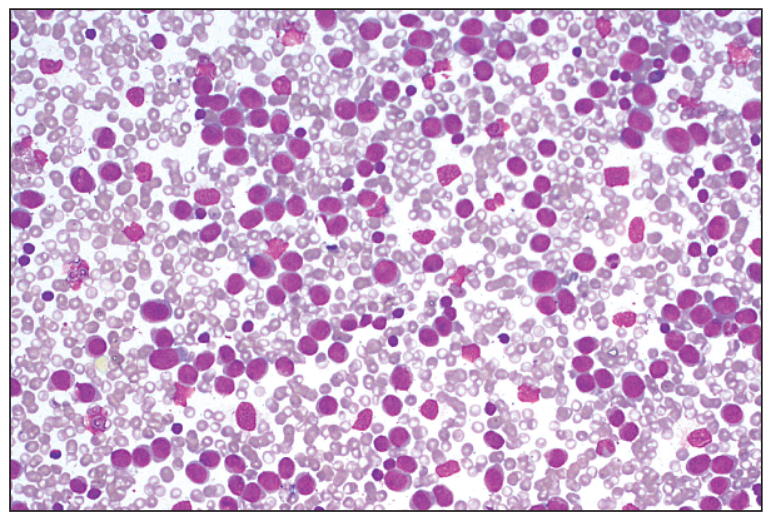

Fig.:-3: Peripheral blood film showing myeloblast

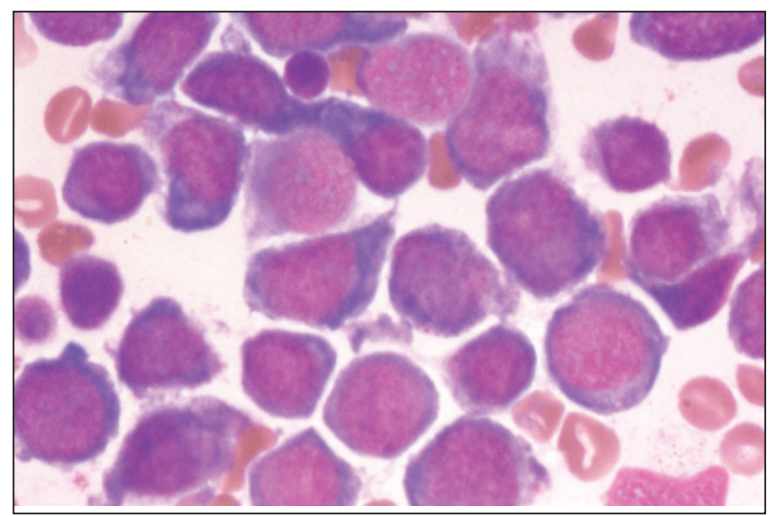

Fig-4 Smear of aspirated bone marrow showing stained with Leishman's and magnified at $x 40$ myeloblast stained with Leishman's and magnified at x 100
The parents were counseled about the nature and prognosis of the disease. Then, treatment was started with chemotherapeutic agents according to protocol. After proper hydration, having normal renal and liver function test, intrathecal (methotrexate12.5 mg + Hydrocortisone $25 \mathrm{mg}$ ) on day 1, Donorubicin 30 $\mathrm{mg} / \mathrm{m}^{2}$ intravenously on day 1 and day 2, Cytarabine $100 \mathrm{mg} / \mathrm{m}^{2}$ intravenously over one hour on day 1 to day 7 and Etoposide $100 \mathrm{mg} / \mathrm{m}^{2}$ intravenously over one hour on day 4 and day 5 was started. On the second day of chemotherapy on $6^{\text {th }}$ September, 2007 her vital sign suddenly deteriorated and the baby expired on $8^{\text {th }}$ September 2007, may be due to leukostasis or intracranial hemorrhage.

\section{Case Report-2:}

Chayon, a nine month old female baby weighing $8 \mathrm{~kg}$ hailing from Natore, Bangladesh was admitted in Pediatric Hematology and Oncology, BSMMU on $25^{\text {th }}$ August, 2007 with the history of fever, proptosis of both eye ball, deviation of the angle of mouth to right side and unable to close the left eye for one month (Fig-5).

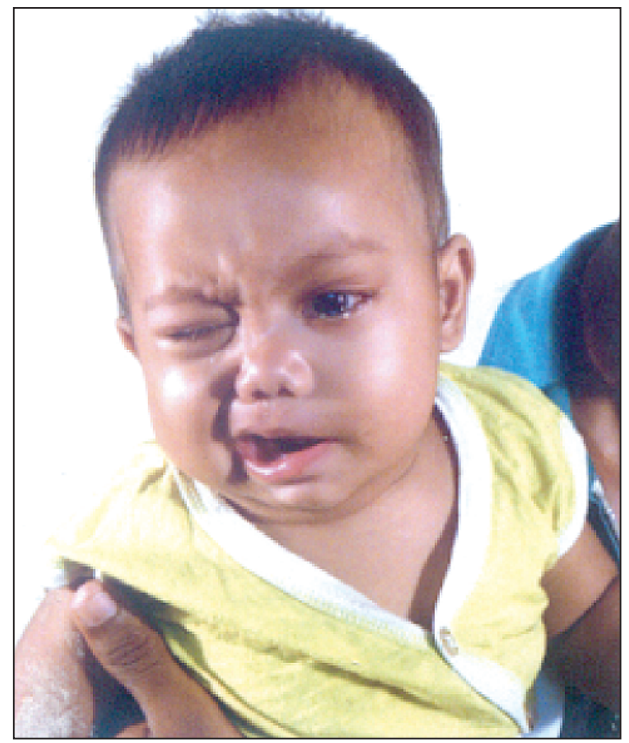

Fig.-5: Photograph (facial palsy) at presentation

The patient came from below average socioeconomic status, completely immunized. There was no history of cough, respiratory distress, vomiting, any bleeding manifestation or ear discharge. She was delivered at home at term without any resuscitation. Physical 
examination revealed facial asymmetry with the features of left sided facial nerve palsy along with proptosis of both eyeballs. She was moderately pale, oral cavity normal, moderate hepatosplenomegaly, skin survey revealed normal. Hematological investigations showed the followings: hemoglobin level $5.8 \mathrm{gm} / \mathrm{dl}$, ESR $60 \mathrm{~mm}$ in $1^{\text {st }}$ hour, total WBC $120 \times 10^{9} / \mathrm{L}$, platelet count $50 \times 10^{9} / \mathrm{L}$, neutrophil $10 \%$, lymphocytes $20 \%$, myeloblasts $70 \%$. Peripheral blood film showed anisochromia with anisocytosis, white blood cell showed shift to the left with the prominamce of myeloblasts, platelets were reduced. CSF study showed the presence of leukemic cell. Bone marrow morphology and cytochemistry were as follows: hypercellular marrow with increased myeloid erythroid ratio. Erythopoesis was hyperplastic with more than $30 \%$ myeloblast. Megakaryocytes were depressed. Cytochemical staining of the aspirated marrow showed PAS negative, MPO positive and diagnosed as AML-M . $_{\text {. }}$ (Fig-6).

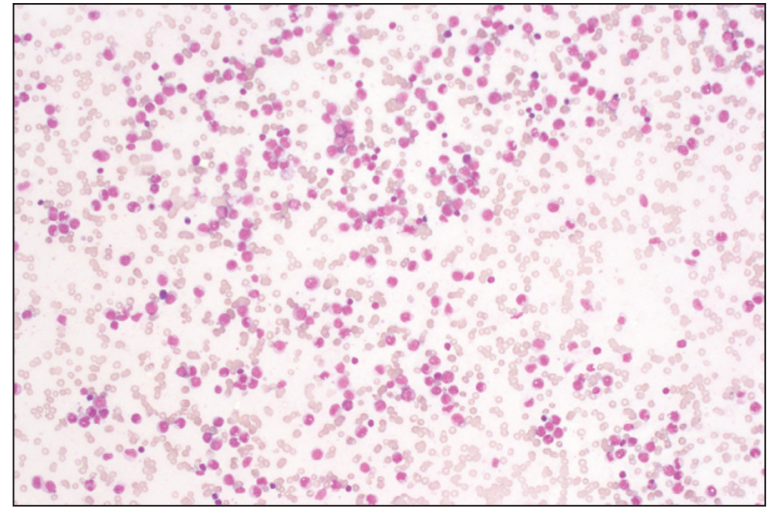

Fig:-6 Smear of aspirated bone marrow showing myeloblast stained with Leishman's and magnified at $x 40$

Having normal liver and renal function test, the parents were counseled about the disease. Then, protocol based first induction chemotherapy was started with intrathecal (methotrexate $12.5 \mathrm{mg}+$ Hydrocortisone $25 \mathrm{mg}$ ) on day 1, Donorubicin 30 $\mathrm{mg} / \mathrm{m}^{2}$ intravenously on day 1 and day 2 , Cytarabine $100 \mathrm{mg} / \mathrm{m}^{2}$ intravenously over 1 hour on day 1 to day 7 , Etoposide $100 \mathrm{mg} / \mathrm{m}^{2}$ intravenously over 1 hour on day 4 and day 5. Gradually, her facial nerve palsy was improved along with the hematological parameters.
Following one month of first induction repeat bone marrow aspiration was performed. The findings were in complete remission along with significant improvement of her facial nerve palsy. Then, second induction therapy was given with same drug but with fine-tuning. After 4 weeks, a consolidation therapy was given with Cytarabine $1 \mathrm{gm}$ intravenously every 12 hourly in day 1 , day 2 and day 3. Repeat bone marrow aspiration findings and hematological parameters were in complete remission. Now, the baby is on continuation phase with Cytarabine $40 \mathrm{mg}$ / $\mathrm{m}^{2}$ intravenously 4 days in a week, Adriamycin 25 $\mathrm{mg} / \mathrm{m}^{2}$ intravenously on day 1 and Thioguanine 40 $\mathrm{mg} / \mathrm{m}^{2}$ orally. Till now, the baby is on regular follow up in our out patient department for last 9 month having significant improvement of her general condition including facial asymmetry along with hematological remission.

\section{Discussion:}

In our first case, GS had association prior to diagnosis of AML and subsequently the child died on the second day of chemotherapy. These findings were consistent with observation of Cavdar et al., 1990 ${ }^{12}$, when they showed that GS and its association with AML are well known entities. Most of the patients are children and young adults. Generally, survival of the cases with GS is shorter than that of the patients without GS who received the same treatment. GS are most commonly found in the orbit or periorbital areas where they can cause ptosis. They may also involve the spinal cord and can cause cauda equine syndrome or paraparesis reported by Bulas et al., $1995^{13}$ and Uyesugi et al., 20007. But in contrast to their findings our observation was that GS found in the auditory canal resulting facial nerve palsy.

GS can precede the bone marrow involvement by just a few weeks to 1 or 2 years reported by Meis et al. $1986^{3}$. Our observations was also consistent with the findings of Meis et al. Involvement of CNS which occur in approximately $2 \%$ of patients that can be in the form of leukemic cells in CSF or chloromas from which patients can present with headache, nausea, vomiting, photophobia, papiledema and cranial nerve palsy, a study performed by Almadori et al. $1996^{14}$. We disagreed the observations of Almadori, et al. though CSF was free from blast cell in our first case 
but there was GS in the auditory canal subsequently developed facial nerve palsy. In both of our patients, facial nerve palsy preceded the diagnosis of AML nearly one month earlier and tumor mass in the auditory canal causing facial nerve palsy one week earlier. After the detection of GS, peripheral blood smear and bone marrow aspiration findings revealed the diagnosis of AML. It could not be established whether there was AML initially, because the evaluation of peripheral blood smear and bone marrow aspiration were not performed earlier in both cases. These findings were consistent with the observations of Chen et al. $1996^{15}$, when they showed that CNS symptoms may occur due to GS in patients with AML. In our first case, CSF study was absence of leukemic cell at presentation but blast cell was present in CSF in second case. After the detection of facial nerve palsy, peripheral blood smear and bone marrow aspiration findings revealed the diagnosis of AML.

AML has a strong association with GS. Rarely, the tumor may be seen before the diagnosis of leukemia. Symptomatic facial nerve palsy by GS is also relatively uncommon, a study performed by Bulent et al,. 2001. ${ }^{16}$ But in our first case, the GS was seen before the diagnosis of AML.

Symptomatic facial nerve involvement in AML patient is rare reported by Kurabayashi et al, 1989. ${ }^{7}$ and Chapman et al., $1980 .^{10}$ It may be due to leukemic cell infiltration at any site of the facial nerve or occurrence of GS in temporal bone or auditory canal and may be accompanied by hearing loss an observation of Kurabayashi et al,. 1989. ${ }^{9}$ But in our second case had only facial nerve palsy due to leukemic cell infiltration without any association of hearing loss.

The treatment of AML patients with facial nerve palsy is chemotherapy/or radiotherapy, an observation of Chapman et al.,1980. ${ }^{10}$ Though unfortunately we could not treat with chemotherapeutic agents with proper duration in our first case, as she succumbed after the second day of chemotherapy may be due to suspected intracranial hemorrhage or leukostasis.

But in our second case we have given a protocol based induction of remission with chemotherapeutic agents having leukemic cells infiltration in CSF. Second bone marrow aspiration and other hematological investigations were done subsequently. Her physical condition along with the facial nerve palsy was improved gradually and bone marrow aspiration findings were in complete remission. Till now, the baby is on maintenance therapy for last nine month with regular follow up in our out patient department with significant improvement of her facial nerve palsy including hematological remission.

\section{Conclusion:}

So, an awareness of varied clinical manifestation of childhood AML is needed in order to establish the correct diagnosis when the presenting signs and symptoms are enigmatic. As in these cases, patients may get admitted in the hospital with isolated facial nerve palsy before the diagnosis of childhood AML. GS and leukemic cell infiltration should be considered in the differential diagnosis, particularly, in the pediatric patients presenting with isolated facial nerve palsy. Complete blood count along with peripheral blood film examination should be done routinely and if atypical cell are found then bone marrow aspiration and morphological examination should be done to establish the diagnosis. In patients with GS and facial nerve palsy, chemotherapy and/or radiotherapy should be tried prior to surgery because of the good response.

\section{References:}

1. Dock G. Chloroma and its relation to leukemia. Am J Med Sci 1893; 106: 152-57.

2. Wiernik PH, Serpick AA. Granulocytic sarcoma (chloroma). Blood 1970; 35: 361-69.

3. Meis JM, Butler JJ, Osborne BM, Manning JT. Granulocytic sarcoma in nonleukemic patients. Cancer 1986; 58: 2697-709.

4. Dusenbery KE, Howells WB, Arthur DC, et al. Extra medullary leukemia in children with newly diagnosed acute myeloid leukemia: a report from the Children Cancer Group. J Pediatr Hematol Oncol 2003; 25 : 760-68.

5. Neiman RS, Barcos M, Berard C, Mann R, Rydell RE, Bennet JM. Granulocytic sarcoma: a clinicopathologic study of 61 biopsied cases. Cancer 1981; 48: 1426-137.

6. Krause JR. Granulocytic sarcoma preceding acute leukemia: a report of six cases. Cancer 1979; 44: 1017-21.

7. Uyesugi WY, Watabe J, Petermann G. Orbital and facial granulocytic sarcoma (chloroma): a case report. Pediatr Radiol 2000; 30: 276-78 
8. Doshi HM, Schochet SS, Gold M, Nugent GR. Granulocytic sarcoma presenting as an epidural mass with acute parapareses in an aleukemic patient. Am J Clin Pathol 1991; 95: 228-32.

9. Kurabayashi H, Miyawaki S, Naruse T, Kubota K. Bilateral tympanic cavity infiltrtation with effusion in a patient with acute myeloblastic leukemia. Blut 1989; 58: 45-46.

10. Chapman P, Johnson SA. Mastoid chloroma as relapse in acute myeloid leukemia. J Laryngol Otol 1980; 94: 1423 27.

11. Lilleyman JS. Neurological complications of acute childhood leukemia. J Royal Soc Med 1993; 86: 252-53.

12. Cavdar AO, Babacan E, Gozdasoglu S, Kilicturgay K, Arcasoy A, Cin S, et al. High risk subgroup of acute myelomonocytic leukemia (AMML) with orbito-ocular granulocytic sarcoma (OOGS) in Turkish Children: retrospective analysis of clinical, hematological, ultrastructral and therapeutic findings of thirty three OOGS Acta Haematol 1990; 83: 89-93.

13. Bulas RB, Laine FJ, Das NL. Bilateral orbital granulocytic sarcoma (chloroma) preceding the blast phase of acute myelogenons leukemia: CT findings. Pediatr Radiol 1995; 25: 488-89

14. Almadori G, Del NM, Cadoni G, et al. Facial nerve paralysis in acute otomastoidits as presenting symptoms of FABM2, T8, 21 leukemic relapse. Case report and review of literatures. Int J Pedi Orthinolargyngol. 1996; 36: 45-52.

15. Chen CY, Zimmerman RA, Faro S, Bilaniuk LT, Chou TY, Molloy PT. Childhood leukemia: central nervous system abnormalities during and after treatment. Am J Neuroradiol 1996; 17: 295-310

16. Bulent E, Mustafa C, Olgum K, Ali U, Ozlem Er, Hasan SC and Mustafa A. Facial nerve palsy and paraplegia as presenting symptoms of acute myeloid leukemia. Jpn J Clin Oncol 2001; 31:86-88. 